\title{
Определение параметров структур металл-диэлектрик-полупроводник со сверхтонким изолирующим слоем из высокочастотных вольт-фарадных характеристик
}

\author{
(C) Е.И. Гольдман, Н.Ф. Кухарская, С.А. Левашов, Г.В. Чучева \\ Фрязинский филиал Института радиотехники и электроники им. В.А. Котельникова \\ Российской академии наук, \\ 141190 Фрязино, Россия \\ E-mail: gvc@ms.ire.rssi.ru
}

(Получена 14 декабря 2017 г. Принята к печати 29 декабря 2018 г.)

\begin{abstract}
Предложена простая численная методика обработки данных высокочастотных вольт-фарадных характеристик структур металл-диэлектрик-полупроводник. Подход основан на анализе экспериментальных характеристик вблизи состояния плоских зон, где перезарядка поверхностных локализованных электронных состояний малосущественна по сравнению с изменением приграничного заряженного слоя в полупроводнике. Развитый аппарат позволяет: во-первых, находить необходимые параметры полупроводника и изолирующего слоя; во-вторых, получать в диапазоне от плоских зон до глубокого обеднения экспериментальные полевые зависимости изгиба зон в полупроводнике и суммарной концентрации встроенного заряда, заряда пограничных состояний и неосновных носителей заряда на границе раздела полупроводник-диэлектрик. Методика хорошо применима к структурам со сверхтонким изолирующим слоем. На образцах металл-окисел-полупроводник на основе $n$-Si с толщиной окисла $39 \AA$ проведена экспериментальная апробация предложенного подхода. Точность полученных результатов составила $2-3 \%$.
\end{abstract}

DOI: $10.21883 /$ FTP.2019.01.46985.8802

Экспериментальные исследования процессов, происходящих в изолирующих слоях и на их границах раздела (ГР) с полупроводником, требуют контроля изменений потенциального и зарядового рельефов в структурах металл-диэлектрик-полупроводник (МДП). Информацию об изгибе зон в полупроводнике, заряде пограничных состояний и накоплении неосновных носителей заряда у поверхности получают из результатов измерений высокочастотной емкости МДП структур на базе классических выражений для потенциального рельефа [1]. Для обработки данных высокочастотных вольт-фарадных характеристик (ВФХ) необходимо знать значения емкости изолирующего слоя, $C_{i}$, площади полевого электрода, $S$, и концентрации легирующей примеси в полупроводнике непосредственно у его границы с диэлектриком, $N_{d}$. Первые две величины находятся достаточно легко: $C_{i}$ совпадает с максимальным значением высокочастотной емкости $\bar{C}$, отвечающей поверхностному заряженному слою полупроводника и изолирующему промежутку (т.е. в области глубокого обогащения). Переменная $\bar{C}$ как функция напряжения полевого электрода $V_{g}$ определяется непосредственно по данным измерений (см. далее). Значение $S$ устанавливается из оптических измерений. Сложнее с величиной $N_{d}$. Во-первых, например, для кремниевых структур известно [2], что в процессах высокотемпературного окисления поверхности $\mathrm{Si}$ и очистки получаемых структур концентрация $N_{d}$ у ГР $\mathrm{Si}-\mathrm{SiO}_{2}$ может измениться по сравнению с ее значением в толще полупроводниковой подложки. Эти отклонения могут достигать десятков процентов, что для структур со сверхтонким изолирующим слоем будет критически сказываться на резуль- татах обработки экспериментальных ВФХ. Во-вторых, в опытах при комнатной температуре пограничные состояния перезаряжаются практически вплоть до состояния плоских зон полупроводника. Поэтому, как будет показано далее, использование часто применяемого представления ВФХ в модели слоя обеднения Шоттки с не зависящим от напряжения встроенным зарядом будет приводить к значительным погрешностям. Таким образом, значение концентрации легирующей примеси в полупроводнике непосредственно у его границы с диэлектриком и рассчитываемые на его основе величины безразмерных параметров структуры необходимо вычислять из данных измерений полевых зависимостей высокочастотных емкостей МДП структур. Проводить расчеты нужно в окрестности состояния плоских зон, в области, где пограничные состояния уже практически заполнены и почти не изменяют свой заряд с ростом полевого напряжения. При этом опасно переходить к состоянию сильного обогащения, где эффекты вырождения и размерного квантования электронной системы приводят к существенным отклонениям [3] вида ВФХ от классического [1]. Развитию такого подхода к определению значений параметров МДП структур из экспериментальных высокочастотных ВФХ и посвящена данная работа.

Специфика образцов со сверхтонким диэлектриком, с толщиной слоя $h<50 \AA$, заключается в необходимости как минимум учета сопротивления базы при измерении ВФХ [4-6] и проведении опытов в неповреждающих условиях, т.е. в ограниченном диапазоне напряжений и за достаточно короткие времена $[7,8]$. Величины емкости $\bar{C}$ и проводимости полупроводниковой подлож- 
ки $\rho_{b}$ определяются через измеренные при двух высоких частотах значения емкости МДП структуры $C_{1}$ и $C_{2}$ по формулам $[4,5]$

$$
\begin{gathered}
\bar{C}=\frac{C_{1} C_{2}\left(\omega_{1}^{2}-\omega_{2}^{2}\right)}{\omega_{1}^{2} C_{1}-\omega_{2}^{2} C_{2}}, \\
\rho_{b}=\frac{\sqrt{\left(C_{2}-C_{1}\right)\left(\omega_{1}^{2} C_{1}-\omega_{2}^{2} C_{2}\right)}}{C_{1} C_{2}\left(\omega_{1}^{2}-\omega_{2}^{2}\right)},
\end{gathered}
$$

где $\omega_{1}$ и $\omega_{2}$ - циклические частоты изменения тестирующего напряжения.

Изгиб зон в полупроводнике $n$-типа проводимости, $V_{s}$, и выраженная в см$^{-2}$ суммарная концентрация встроенного заряда, заряда пограничных состояний и дырок на $\Gamma \mathrm{P}, p_{s q}$, связаны с емкостью $\bar{C}$ классическими соотношениями [1], которые в безразмерной форме имеют вид

$$
\begin{aligned}
& \frac{\bar{C}}{C_{i}-\bar{C}}=\left(\frac{q V_{s h}}{T}\right)^{1 / 2} \frac{\left|1-e^{-v_{s}}\right|}{\left(e^{-v_{s}}+v_{s}-1\right)^{1 / 2}}, \\
p_{s q}= & -\frac{C_{i} T}{S q}\left[\frac{q V_{g}}{T}+v_{s}\right. \\
& \left.+2\left(\frac{q V_{s h}}{T}\right)^{1 / 2}\left(e^{-v_{s}}+v_{s}-1\right)^{1 / 2} \operatorname{Sgn} v_{s}\right],
\end{aligned}
$$

где $v_{s}=-q V_{s} / T-$ безразмерный изгиб зон в полупроводнике $\left(v_{s}>0\right.$ при обеднении и $v_{s}<0$ при обогащении; если не учитывать неравенство $\left.V_{g}\right|_{v_{s}=0} \neq 0$, то область $V_{g}<0$ отвечает обеднению полупроводника, а $V_{g}>0$ - обогащению), $q$ - элементарный заряд, $T$ - температура в энергетических единицах, $V_{s h}=2 \pi \varkappa_{s} q N_{d} h^{2} / \varkappa_{i}^{2}, \varkappa_{s}$ и $\varkappa_{i}$ - диэлектрические проницаемости полупроводника и изолятора соответственно, $h$ - толщина окисла. ${ }^{1}$

Формула (2) справедлива и в случае перезарядки локализованных электронных состояний. Следовательно, для установления из равенств (2) и (3) экспериментальных зависимостей $v_{s}\left(V_{g}\right)$ и $p_{s q}\left(V_{g}\right)$ необходимо знать значение параметра $\left(q V_{s h} / T\right)$.

В окрестности плоских зон должен существовать интервал напряжений $V_{g}$, где величина $p_{s q}$ практически не изменяется. В этой области, дифференцируя по $V_{g}$ выражения (2) и (3), получаем

$$
\left(\frac{C_{i}}{C_{i}-\bar{C}}\right)^{2} \frac{2}{\bar{C}} \frac{T d \bar{C}}{q d V_{g}}=\frac{\left(e^{-2 v_{s}}+2 v_{s} e^{-v_{s}}-1\right)}{\left(e^{-v_{s}}+v_{s}-1\right)\left|1-e^{-v_{s}}\right|} .
$$

Удобство соотношения (4) для вычисления параметра $\left(q V_{s h} / T\right)$ заключается в прямой связи левой части, определяемой из экспериментальных данных, с правой частью, являющейся функцией только изгиба зон. Теперь процедуру определения значения параметра $\left(q V_{s h} / T\right)$ можно провести, используя последовательные прибли-

\footnotetext{
${ }^{1}$ Следует отметить, что в структурах $\mathrm{Si}-\mathrm{SiO}_{2}-$ поли-Si co сверхтонким окислом переходные слои могут занимать более $40 \%$ объема изолятора. Поэтому фактически значения $\varkappa_{i}$ и $h$ должны быть эффективными и зависеть от толщины изолятора и технологии его приготовления.
}

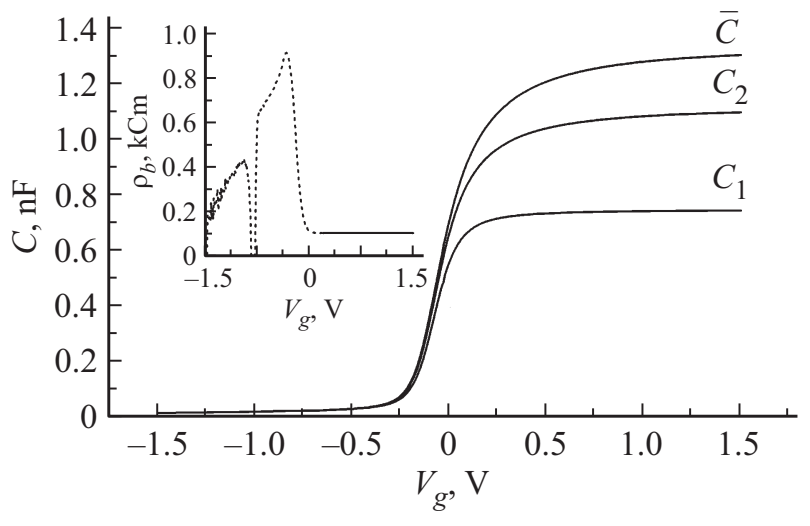

Рис. 1. Зависимости $C_{1}\left(V_{g}\right), C_{2}\left(V_{g}\right)$ и $\bar{C}\left(V_{g}\right)$, $C_{i}=1.303 \cdot 10^{-9} \Phi$. На вставке - зависимость $\rho_{b}\left(V_{g}\right)$. Пунктирной линией показана область превышения точности измерений.

жения. На первом шаге, полагая $v_{s} \rightarrow 0$, из выражения (4) находим напряжение $V_{g}$, соответствующее плоским зонам. По значению емкости $\bar{C}$, отвечающему этому напряжению, из соотношения (2) определяем величину параметра $\left(q V_{s h} / T\right)$. Используя его из формулы (2), строим зависимость $v_{s}\left(V_{g}\right)$, а из выражения (3) функцию $p_{s q}\left(V_{g}\right)$. Определяем напряжение $V_{g}$, отвечающее центру плато на зависимости $p_{s q}-V_{g}$, а уже по зависимости $v_{s}\left(V_{g}\right)$ - значение $v_{s}$ в центре плато. ${ }^{2}$ С этим значением $v_{s}$ переходим ко второму шагу: из выражения (4) определяем напряжение $V_{g}$, ему соответствующее; из формулы (2) находим новую величину параметра $\left(q V_{s h} / T\right)$; из соотношений (2), (3) строим новые зависимости $v_{s}\left(V_{g}\right)$ и $p_{s q}\left(V_{g}\right)$; по центру плато функции $p_{s q}\left(V_{g}\right)$ получаем новое значение $v_{s}$ и с ним переходим к следующему шагу. Процедура повторяется, пока разница значений параметра $\left(q V_{s h} / T\right)$ не окажется в пределах требуемой точности.

Экспериментальная апробация предложенной методики была проведена при исследованиях МOП $n$-Si-cтруктуры с толщиной окисла $39 \AA$ ( $h$ определена из оптических измерений) и площадью полевого электрода $1.6 \cdot 10^{-3} \mathrm{~cm}^{2}$. Измерения ВФХ проводились на частотах $1\left(C_{1}\right)$ и $0.5 \mathrm{MГц}\left(C_{2}\right)$ МГц на компьютеризованной экспериментальной установке $[9,10]$ с использованием прецизионного измерителя LCR Agilent E4980A в динамическом режиме: $V_{g}$ изменялось со скоростью полевой развертки $\beta=16 \mathrm{MB} / \mathrm{c}$ вначале от 1.5 до $-1.5 \mathrm{~B}$ (прямая ветвь), затем от -1.5 до $1.5 \mathrm{~B}$ (обратная ветвь). Зависимости $C_{1}\left(V_{g}\right), C_{2}\left(V_{g}\right)$ и $\bar{C}\left(V_{g}\right)$ представлены на рис. 1 ; прямая и обратная ветви практически совпадают; они различимы только в области генерации дырок $[7,8]$.

\footnotetext{
${ }^{2}$ Под плато следует понимать отрезок графика в области обеднения полупроводника, где величина $p_{s q}$ изменяется с напряжением максимально слабо. По крайней мере должно быть выполнено условие $q\left|d p_{s q} / d V_{g}\right| \ll\left|d Q_{s d} / d V_{g}\right|$, где $Q_{s d}=\left(2 C_{i} T / q S\right)$ $\times\left(q V_{s h} T\right)^{1 / 2}\left(e^{-v_{s}}+v_{s}-1\right)^{1 / 2} \operatorname{Sgn} v_{s}$ - заряд единицы площади поверхности полупроводника, связанный с перетеканием свободных электронов при изменении полевого напряжения.
} 
Значения напряжений $V_{g}$, принимаемые для расчета уравнения (4), безразмерного изгиба зон $v_{s}$ и параметра $\left(q V_{s h} / T\right)^{1 / 2}$ для каждой итерации

\begin{tabular}{c|c|c|c}
\hline $\begin{array}{c}\text { Номер } \\
\text { итерации }\end{array}$ & $V_{g}, \mathrm{~B}$ & $v_{s}$ & $\left(q V_{s h} / T\right)^{1 / 2}$ \\
\hline 2 & -0.245 & 1.511 & $7.323 \cdot 10^{-2}$ \\
3 & -0.235 & 1.158 & $7.323 \cdot 10^{-2}$ \\
4 & -0.224 & 0.723 & $7.280 \cdot 10^{-2}$ \\
5 & -0.221 & 0.622 & $7.258 \cdot 10^{-2}$ \\
6 & -0.218 & 0.483 & $7.213 \cdot 10^{-2}$ \\
7 & -0.213 & 0.332 & $7.286 \cdot 10^{-2}$
\end{tabular}

На вставке к рис. 1 показано поведение проводимости полупроводниковой подложки при изменении полевого напряжения. Отклонение от постоянного значения происходит только в области, где из-за слияния кривых $C_{1}\left(V_{g}\right)$ и $C_{2}\left(V_{g}\right)$ разность $\left(C_{2}-C_{1}\right)$ становится меньше точности измерений. Максимальное значение $\bar{C}$ составило $1.303 \cdot 10^{-9} \Phi$; оно и было принято за величину $C_{i}$. Как и следовало ожидать (см. сноску ${ }^{1}$ ), рассчитанная по данному значению $C_{i} \mathrm{c}$ величиной диэлектрической проницаемости окисла, соответствующей массивному образцу, толщина окисла оказалась $h=42.4 \AA$, что несколько больше $h$, полученной из оптических измерений.

В соответствии с ранее изложенной методикой было сделано 7 последовательных приближений. На рис. 2 и 3 показаны соответствующие кривые $v_{s}\left(V_{g}\right)$ и $p_{s q}\left(V_{g}\right)$. На первом шаге (кривые 1 ) было выбрано значение $v_{s}=0$, ему в соотношении (4) отвечала величина $V_{g}=-0.202 \mathrm{~B}$, а из выражения (2) получен параметр $\left(q V_{s h} / T\right)^{1 / 2}=7.413 \cdot 10^{-2}$. Данные, выбираемые далее для расчетов, а также получающиеся результаты для переменной $v_{s}$ и параметра $\left(q V_{s h} / T\right)^{1 / 2}$ приведены в таблице; на рис. 2 и 3 им соответствуют кривые 2-7. Для последней итерации в окрестности избранного напряжения $V_{g}$ удельные емкости составили: $q\left|d p_{s q} / d V_{g}\right| \approx 9.4 \cdot 10^{-9} \Phi / \mathrm{cm}^{2}$, $\left|d Q_{\mathrm{sd}} / d V_{g}\right| \approx \frac{\bar{C}}{S}=6.6 \cdot 10^{-8} \Phi / \mathrm{cm}^{2}$. Из рис. 2,3 и таблицы следует, что процедура расчетов - не расходящаяся, выбираемые для вычислений значения напряжений и получающиеся величины переменной $v_{s}$, параметра $\left(q V_{s h} / T\right)$ сгущаются в малых окрестностях вокруг своих средних значений, последовательность повышения и понижения кривых $p_{s q}\left(V_{g}\right)$ в результате итераций повторяется через 6 шагов. Значения параметра $\left(q V_{s h} / T\right)$ на последних итерациях изменяются только во втором знаке после запятой. Отсюда следует оценка погрешности в 2-3\%. По значению параметра $\left(q V_{s h} / T\right)$ на последнем шаге можно определить величину концентрации легирующей примеси в полупроводнике непосредственно у его границы с диэлектриком. Если принять для диэлектрической проницаемости окисла значение, соответствующее массивному образцу (см. сноску ${ }^{1}$ ), получается $N_{d}=1.117 \cdot 10^{15} \mathrm{~cm}^{-3}$.

Отметим, что часто используемая методика [1] определения концентрации легирующей примеси по наклону прямолинейного участка $\bar{C}^{-2}\left(V_{g}\right)$ в области $v_{s} \gg 1$ для высокочастотных измерений образцов со сверхтонким изолирующим слоем при комнатных температурах не работает. На рис. 4 зависимость $\bar{C}^{-2}\left(V_{g}\right)$ приведена, и экспериментальная линия представляет собой практически отрезок прямой. Вычисленное из наклона этой прямой

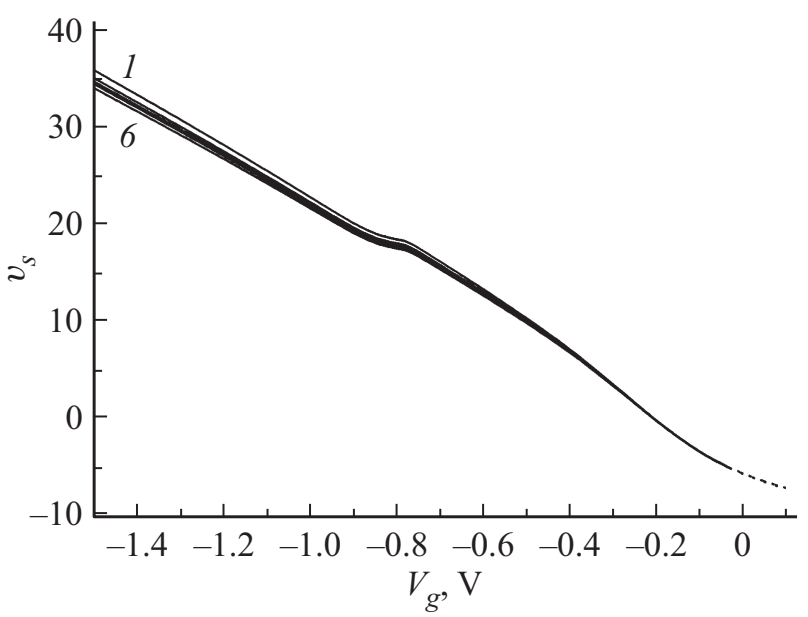

Рис. 2. Зависимости безразмерного изгиба зон $v_{s}$ от полевого напряжения $V_{g}$ для каждого приближения. $(1-7)-$ номера итераций, из-за близости кривых показаны номера только самой верхней (1) и самой нижней (6) кривых. В области обогащения линии даны пунктиром в связи с несоответствием применяемого классического аппарата условиям вырождения и пространственного квантования.

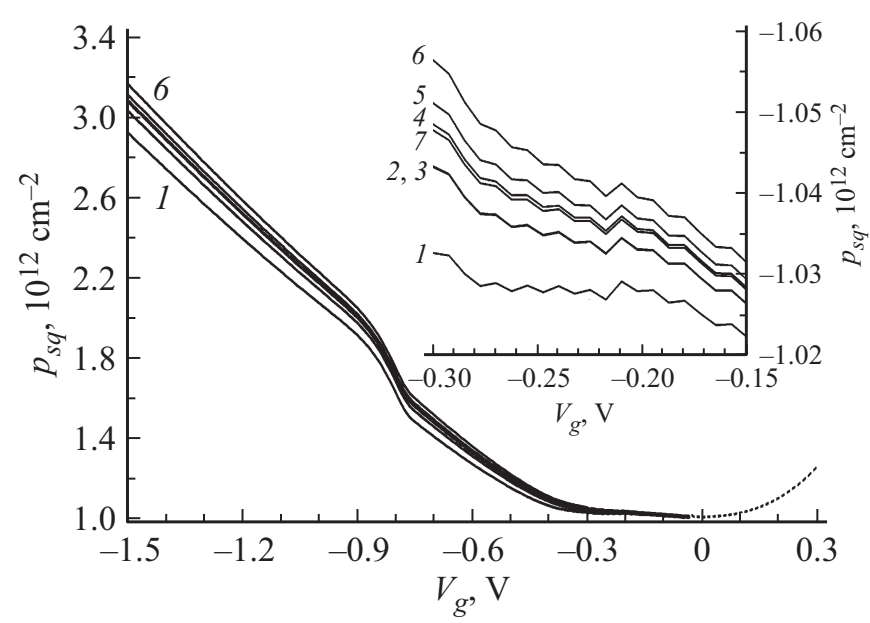

Рис. 3. Зависимости $p_{s q}\left(V_{g}\right)$ для каждой итерации. $(1-7)-$ номера итераций, из-за близости кривых показаны номера только самой верхней (6) и самой нижней (1) кривых. Пунктирная линия - см. подпись к рис. 2. На вставке увеличенный фрагмент кривых в области напряжений, где проводились расчеты параметра $\left(q V_{s h} / T\right)$. 


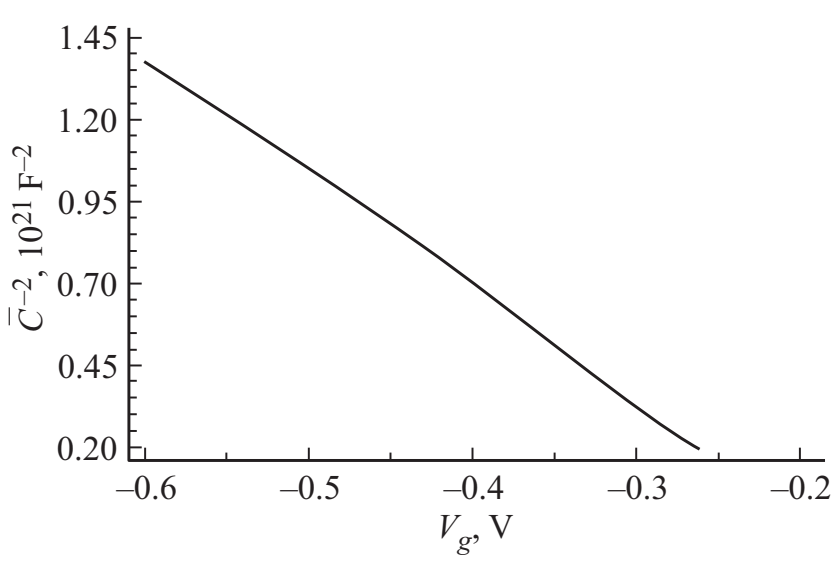

Рис. 4. Зависимость $\bar{C}\left(V_{g}\right)$ в координатах Шоттки.

значение $N_{d}=2.2 \cdot 10^{12} \mathrm{~cm}^{-3}$ абсолютно несовместимо с реальными параметрами кремниевых подложек. ${ }^{3}$

Таким образом, представлена простая численная методика обработки данных ВФХ образцов со сверхтонким изолирующим слоем при комнатных температурах. Развитый аппарат позволяет: во-первых, находить необходимые параметры полупроводника и изолирующего слоя; во-вторых, получать в диапазоне от плоских зон до глубокого обеднения экспериментальные полевые зависимости изгиба зон в полупроводнике и суммарной концентрации встроенного заряда, заряда пограничных состояний и неосновных носителей заряда на ГР полупроводник-диэлектрик.

Работа выполнена при частичной финансовой поддержке гранта РФФИ № 16-07-00666 и в рамках Программы фундаментальных исследований президиума РАН „Наноструктуры: физика, химия, биология, основы технологий“.

\section{Список литературы}

[1] S.M. Sze, K.Ng. Kwok. Physics of semiconductor devices, 3rd edn (N.Y, John Willey @ Sons, 2007).

[2] E.H. Nicollian, I.R. Brews. MOS (Metal Oxide Semiconductor) Physics and Technology (N.Y., John Willey @ Sons, 1982).

[3] Т. Андо, А. Фаулер, Ф. Стерн. Электронные свойства двумерных систем (М., Мир, 1985).

[4] L.F. Lonnum, J.S. Johannessen. Electron. Lett., 22 (9), 456 (1986).

[5] J.Y. Kevin, H. Chenming. IEEE Trans. Electron. Dev., 46 (7), 1500 (1999).

[6] Е.И. Гольдман, А.И. Левашова, С.А. Левашов, Г.В. Чучева. ФТП, 49 (4), 483 (2015).

[7] Е.И. Гольдман, Н.Ф. Кухарская, В.Г. Нарышкина, Г.В. Чучева. ФТП, 45 (7), 974 (2011).

\footnotetext{
${ }^{3}$ В этой области происходит сильное изменение суммарной концентрации встроенного заряда, заряда пограничных состояний и неосновных носителей заряда.
}

[8] Е.И. Гольдман, С.А. Левашов, В.Г. Нарышкина, Г.В. Чучева. ФТП, 51 (9), 1185 (2017).

[9] Е.И. Гольдман, А.Г. Ждан, Г.В. Чучева. ПТЭ, № 6, 110 (1997).

[10] Е.И. Гольдман, Н.Ф. Кухарская, В.Г. Нарышкина, Г.В. Чучева. ПТЭ, № 6, 81 (2011).

Редактор Л.В. Шаронова

\section{Determination of parameters of metal-dielectric-semiconductor structures with a ultrathin insulating layer from high-frequency capacitance-voltage measurements}

\section{E.I. Goldman, N.F. Kuharskaya, S.A. Levashov, G.V. Chucheva}

Fryazino Branch of Kotel'nikov Institute of Radioengineering and Electronics, Russian Academy of Sciences, 141190 Fryazino, Russia

\begin{abstract}
The simple numerical technique for processing data of high-frequency capacitance-voltage measurements of metal-insulator-semiconductor structures is proposed. The approach is based on the analysis of experimental characteristics near the state of flat bands, where the overcharge of surface localized electronic states is of little importance compared with variation of the charged boundary layer in the semiconductor. The developed apparatus allows: first, to find necessary parameters of a semiconductor and an insulating layer; secondly, to obtain in the range from flat bands to the deep depletion region experimental field dependences of the band bending in the semiconductor and the total concentration of the built-in charge, the charge of the boundary states, and the minority charge carriers at the semiconductor-insulator interface. The technique is well applicable to structures with a ultrathin insulating layer. Based on $n$-Si metal-oxide-semiconductor samples with the oxide thickness of $39 \AA$, an experimental approbation of the proposed approach was carried out. The accuracy of results was $2-3 \%$.
\end{abstract}

